\title{
Intervenciones públicas de inclusión digital: una aproximación a las dimensiones sociales y políticas de la conectividad*
}

\author{
Public Speeches Digital Inclusion: An Approach \\ to the Social and Political Dimensions of \\ Connectivity
}

Patricia Martínez Coral**

Recibido: 15/10/2013

Aprobado Evaluador Interno: 24/11/2014

Aprobado Evaluador Externo: 12/01/2014

\section{Resumen}

Este artículo explora las dimensiones de la brecha en cuanto al acceso a Internet a través de un recorrido por la evolución de esta tecnología y su impacto socio-político en el marco de la Sociedad de la Información y el Conocimiento, esto con el fin de contrastar las condiciones de exclusión que se presentan en el país y las respuestas ofrecidas por la política sectorial vigente en busca de escenarios alternativos de intervención pública orientados hacia el logro efectivo de la inclusión digital.

\section{Palabras clave:}

difusión tecnológica; brecha digital; intervención pública; sociedad de la información

\begin{abstract}
This article explores the dimensions of the Internet access gap through a review on the evolution of this technology and its socio-political impact within the framework of the information and knowledge society, this in order to contrast the conditions of exclusion arising in the country and the answers offered by the current sectorial policy searching alternative scenarios of public intervention oriented to the effective achievement of digital inclusion.
\end{abstract}

\section{Keywords:}

technological diffusion; digital divide; public intervention; information society

doi:10.11144/Javeriana.PAPO19-1.ipid

${ }^{*}$ Artículo de Reflexión. Este artículo es resultado de una reflexión preliminar del objeto de investigación de tesis doctoral.

** Magíster en Gobierno y Políticas Públicas y Profesional en Gobierno y Relaciones Internacionales -Universidad Externado de Colombia. Docente Universidad Externado de Colombia. Correo electrónico: patricia.martinez@uexternado.edu.co. 



\section{Intervenciones públicas de inclusión digital: una aproximación a las dimensiones sociales y políticas de la conectividad.}

Desde finales de los años setenta, las Tecnologías de la Información y la Comunicación, (TIC), han impulsado la emergencia de un nuevo orden social caracterizado por la creciente importancia de la información en cada uno de los aspectos que rige la vida contemporánea. Los desarrollos teóricos que reflejan dichas transformaciones se clasifican en dos vertientes principales; la primera se enmarca en el concepto de Sociedad de la Información que integra múltiples dimensiones a través de las cuales se expresan la centralidad de la tecnología y el valor de la información en las actividades humanas, el sistema económico, y la esfera cultural. Entre los exponentes destacados de este enfoque se encuentran Toffler (1981), Masuda (1984), Bell (1991), Castells (2002) y Baudrillard (2007). La segunda vertiente corresponde a la categoría de análisis denominada Sociedad del Conocimiento, la cual profundiza en el valor de las construcciones logradas a partir del uso de la información. Algunos abordajes representativos son los de Melody (1990), Stehr (1994), Steinmueller (2000) y Mansell (2009).

Grosso modo, la revolución de las TIC consiste en el crecimiento exponencial de las funciones de transmisión, comunicación, procesamiento y almacenamiento de información que ha sido promovido por las mejoras en los instrumentos de cómputo - hardware y software- y los avances en las telecomunicaciones. Sin duda, gran parte de esos adelantos se atribuyen al perfeccionamiento de la Internet que Benkler (200o) define como una red de redes que permite transportar señales de vídeo, voz y datos, y que se organiza de manera vertical a través de la integración de los siguientes niveles funcionales: el nivel de acceso (infraestructura de conectividad), el nivel lógico (interface entre los dispositivos conectados a la red y la infraestructura de acceso, la cual define las secuencias requeridas para el flujo de la información), el nivel de transporte (codificación en paquetes, convertidos en bits que, mediante protocolos, ordena el flujo de la información y asegura la transmisión), el nivel de aplicaciones (programas construidos sobre la red), y el nivel de contenidos (símbolos e imágenes que toman la forma aceptada por las aplicaciones de la red).

La Internet sorprendió al mundo por su acelerado ritmo de expansión. En 1973, tan sólo cinco años después de haber sido lanzada para conectar a un pequeño grupo de universidades en Estados Unidos bajo la iniciativa de la Agencia de Investigación de Proyectos Avanzados del Departamento de Defensa, la Internet emprendió su fase de internacionalización mediante el diseño de las reglas de transmisión de datos en paquetes - Protocolo de Control de Transmisión (TCP) y Protocolo de Internet (IP). En 1987, tras quedar saturada con la conexión de cerca de 10.000 computadores, dio paso a su etapa de comercialización, la cual fue estimulada rápidamente con la puesta en marcha de la Red Informática Mundial - World Wide Web - (Cohen, 2011). Este periodo coincidió con los procesos de liberalización, desregulación y privatización del sector de 


\section{Patricia Martínez Coral}

las telecomunicaciones promovidos como parte de la globalización. Desde entonces, el mercado de Internet se convirtió en un nicho para la inversión de operadores internacionales atraídos por la búsqueda de ventajas sistémicas tales como la reducción de costos a través de economías de escala en el manejo de tráfico, influencia en la fijación de precios de interconexión, integración con empresas de otros segmentos del mercado, y mayor poder de negociación en escenarios multilaterales (Sarkar et al., 1999).

Asimismo, la telefonía móvil experimentó cambios sustanciales. En 1981 fue lanzada la primera generación (1-G) con el uso de sistemas analógicos que, en poco tiempo, fue remplazada por sistemas digitales con mejoras en la calidad, velocidad y herramientas de programación. De ese modo, en 1992 se creó la segunda generación (2-G) que hizo posible incrementar la velocidad de transmisión de $2.4 \mathrm{kbps}$ a $64 \mathrm{kbps}$ y utilizar mensajes de texto. En 2001 fue implementada la tercera generación (3-G) con mayor número de servicios multimedia (voz, vídeo-llamadas, mensajería instantánea, correos electrónicos y descarga de programas) y una velocidad mínima de transmisión de 200 kbps. A partir de 2010, la comercialización de servicios de cuarta generación (4-G), diseñada para funcionar con base en el protocolo IP a través de módems inalámbricos, teléfonos inteligentes y otros dispositivos móviles con una velocidad cercana a $1 \mathrm{Gbit} / \mathrm{s}$, ha permitido evidenciar la convergencia de la tecnología fija y móvil (Kumar, 2010).

La integración progresiva de servicios de voz, datos y vídeo sobre una misma red condujo a una mayor demanda de la capacidad de transmisión, dando como resultado el paso de la Internet de banda angosta a la Internet de banda ancha (BA). En 1989 el Comité Consultivo Internacional Telegráfico y Telefónico (actualmente Comité de Normalización de las Telecomunicaciones de la Unión Internacional de Telecomunicaciones) introdujo la clasificación de redes de banda ancha para diferenciar las capacidades de transmisión superiores a 155 Mbps de los accesos primarios con velocidades entre 64 Kbps y 2 Mbps (Bouwman y Fijnvandraat, 2006). Actualmente, las redes de acceso con capacidades enmarcadas en la categoría de banda ancha incluyen tecnologías sobre cable (Bucle digital de abonado xDSL, redes híbridas de fibra y cable HFC, fibra óptica FTTx, Comunicaciones por línea eléctrica PLC y Ethernet EFM) y tecnologías inalámbricas (Satelitales, bucles inalámbricos LMDS, micro-ondas WiMax, redes locales inalámbricas WLAN, comunicaciones móviles $3 \mathrm{G}$ y $4 \mathrm{G}$ y televisión digital terrestre TDT).

Por su parte, las mejoras tecnológicas de las telecomunicaciones también han estado acompañadas del incremento de aplicaciones que demandan mayor capacidad de transporte de información. El paso de los servicios de la Web de primera generación hacia los servicios de segunda y tercera generación (Web 2.o y Web 3.0) ha estimulado el surgimiento de comunidades virtuales que asimilan estas plataformas a través de la interacción y la innovación participativa, dejando atrás la navegación pasiva y estática. Las herramientas interactivas han logrado extenderse cada vez más en áreas de salud, 
educación, comercio, entretenimiento y trámites de gobierno en línea. Por esta razón, los requerimientos de velocidad y capacidad en la transmisión de datos convierten a las tecnologías de banda ancha en un instrumento de primera necesidad para acceder a esa variada gama de servicios de la información y la comunicación.

En este sentido, la adecuación de infraestructura constituye un eje de acción prioritario por ser la base de la difusión de Internet (BA), sin que por ello se desconozca la relevancia de los demás eslabones del proceso (asequibilidad del servicio y bienes complementarios, fortalecimiento de capacidades para la apropiación tecnológica, y mecanismos dirigidos a incentivar la demanda y uso productivo de las TIC). Con el propósito de contribuir a la comprensión de la brecha digital y la forma en que la problemática del acceso está siendo abordada en el país, el presente artículo expone en su primer aparte una revisión del impacto social de la difusión de Internet (BA); después sigue una descripción detallada de la brecha de acceso en Colombia y las medidas de adecuación de infraestructura previstas por la política de telecomunicaciones vigente, y cierra con la presentación de una serie de reflexiones en torno a las perspectivas de inclusión digital.

\section{Dimensiones sociopolíticas de la conectividad}

La difusión de la Internet (BA), además de generar diversas oportunidades de crecimiento económico por ser una herramienta de competitividad, ha traído consigo profundos impactos para las sociedades contemporáneas. Uno de ellos se refiere a la renovación de la esfera pública, cambio que Castells (2009) describe como el surgimiento de un escenario político donde la interacción social, instrumentada por las redes de comunicación, moldea valores y creencias, haciendo que las principales fuentes de poder queden supeditadas al funcionamiento de los flujos de información que circulan por las redes locales y/o globales. Otra modificación política soportada en las TIC consiste en las dinámicas de empoderamiento ciudadano asociadas a la aplicación de los modelos de democracia electrónica diseñados para mejorar la relación entre el Estado y el ciudadano. Caldow (2004) destaca los siguientes: el modelo de presencia (aplicaciones de acceso a información), interacción (espacios de opinión y deliberación), transacción (servicios directos, trámites en línea), y transformación (adaptación tecnológica de las instituciones públicas en busca de nuevos y mayores beneficios para el ciudadano).

En un sentido más amplio, la conectividad es entendida como un nuevo desafío para la libertad de expresión. Jack Balkin (2004) plantea que las tecnologías digitales han cambiado las condiciones sociales de la comunicación revaluando por completo las teorías de la libertad de expresión más influyentes del Siglo XX (teorías de la democracia deliberativa). Esto se explica, según el autor, porque dichas tecnologías han demostrado que una verdadera cultura democrática, más que la discusión de asuntos públicos y el funcionamiento de instituciones representativas, es aquella en la cual los individuos tienen 
las mismas oportunidades de participar en las formas de construcción de significados y desarrollo de ideas que los identifican como personas y miembros de una comunidad. A diferencia de las teorías del Siglo XX, que estuvieron rodeadas por la emergencia de los medios masivos de comunicación en un entorno en el que la opinión pública era dirigida por unos pocos grupos dominantes y expresiones no políticas como la cultura popular y la libertad individual se mantuvieron menospreciadas, en la Era de la Internet la libertad de expresión reivindica la posibilidad de acceder a la producción y distribución de la cultura mediante el uso de la tecnología digital.

Consciente de estas transformaciones, previamente discutidas en la Cumbre Mundial sobre la Sociedad de la Información (CMSI) celebrada en Ginebra 2003 y Túnez 2005, la Asamblea General de las Naciones Unidas aprobó la Resolución A/HRC/20/L.13, el 29 de junio de 2012, a través de la cual se reconoce que el acceso a Internet es un derecho humano por ser un medio para ejercer la libertad de opinión y expresión, de conformidad con lo previsto en el Artículo 19 del Pacto Internacional de Derechos Civiles y Políticos (ONU, 2012). Esta declaración, sustentada sobre la base de la autonomía personal y los principios democráticos, comporta obligaciones estatales que se configuran tanto en la promoción del acceso y disponibilidad de la Internet como en las dimensiones del uso de la conectividad referidas a la ausencia de interferencias en el intercambio de información. En complemento de lo anterior, el acceso y uso de la Internet (BA) pueden concebirse, desde el enfoque de las capacidades humanas, como una oportunidad para el ejercicio de libertades enmarcadas en la realización de un estilo de vida digno, valioso y productivo (Birdsall, 2011).

En este contexto, el arribo de la Internet no sólo ha permitido reinterpretar la naturaleza de la tecnología, evidenciando que la relación entre ésta y la sociedad no es unidireccional sino que se nutre de los efectos de su apropiación por parte de individuos y comunidades (DiMaggio et al., 2001) y también ha propiciado incesantes aproximaciones al problema de la brecha digital. Pese a que la formulación conceptual de la brecha digital se dificulta en razón de la innovación tecnológica y los cambios observados en el comportamiento de sus usuarios, es posible identificar el carácter multifacético del término. De acuerdo con Warschauer (2003), la brecha digital combina una dimensión física (referida al servicio de conectividad y acceso a dispositivos electrónicos como computadores y otras terminales) una compuesta por recursos digitales (relacionada con la alfabetización básica y el dominio de lenguas extranjeras que predominan en los contenidos de la red), una asociada a las habilidades y conocimientos indispensables para el manejo de hardware, software y aplicaciones de la red y finalmente una que engloba recursos sociales de los cuales depende el proceso de difusión tecnológica (extensión de las redes familiares, comunitarias, sociales e institucionales).En sus inicios, la noción de brecha digital estuvo ligada a las condiciones de acceso a las TIC y, de manera progresiva, pasó a enfatizar la importancia de su respectivo aprovechamiento en la medida que se 
fueron ampliando repositorios de encuestas y otras fuentes de información acerca del uso de Internet. Así, por ejemplo, al concluir la primera década de difusión de la red, Norris (2001) ilustró cómo la brecha digital constituye un reflejo de las disparidades socio-económicas de carácter endémico tales como el nivel de ingresos, escolaridad y ocupación, entre otros; al mismo tiempo, la brecha se convierte en fuente de nuevas formas de desigualdad entre naciones (brecha global), miembros de una sociedad (brecha nacional), y quienes encuentran la posibilidad de ejercer un rol activo o pasivo en escenarios de participación política provistos por la red (brecha democrática). Una década más tarde, se contempla la necesidad de impulsar transformaciones culturales que contribuyan a una apropiación tecnológica efectiva (Hilbert, 2011).

\section{Brecha de acceso en Colombia y el papel del Estado}

En materia de acceso a las TIC, la brecha que separa a los países industrializados de los países en vía de desarrollo refleja la magnitud del reto que enfrenta la difusión tecnológica. De acuerdo con las estadísticas de la Unión Internacional de Telecomunicaciones, en 2013 el primer grupo de países registra una penetración de suscriptores, por cada 100 habitantes, de 78,4 en el servicio de banda ancha móvil; 27 en el de banda ancha fija; y una penetración por hogar de 77,7\% en el servicio de Internet y 75,5\% en acceso a computador. Por su parte, las suscripciones por cada 100 habitantes en el grupo de países en vía de desarrollo corresponden a un total de 19,8 en el servicio de banda ancha móvil, 6 en el de banda ancha fija, y la penetración de acceso a Internet por hogar es de $28 \%$, mientras que la de computadores es de 27,6\% (ITU, 2013).

A su vez, el Informe Sectorial del Ministerio de Tecnologías de la Información y las comunicaciones (Min TIC) permite evidenciar las brechas de acceso que persisten al interior del país. En el reporte del primer trimestre de 2013 se registró un total de 6.634.659 conexiones a Internet (BA), de las cuales 4.013.553 son fijas y 2.621.106 móviles. Los mayores índices de penetración por suscriptores (sobre el total de la población) son encabezados por Bogotá con 16,45\%, Antioquia con 12,54\%, Risaralda con 11,63\%, Santander con 11,17\%, y Valle 9,9\%. La penetración en departamentos como Córdoba, Cauca, Nariño, Arauca, Chocó, Caquetá, La Guajira y Putumayo no supera el 3\%, y en el caso de los territorios nacionales - Amazonas, Vichada, Vaupés, Guainía y Guaviare - no alcanza el 1\% (Min TIC, 2013). De otro lado, según la Encuesta TIC del DANE, en Colombia 38,4\% de los hogares en 2012 poseía computador de escritorio o portátil con una disparidad entre zonas urbanas y rurales, $46,8 \%$ de los hogares de cabeceras municipales cuentan con equipo, mientras el porcentaje disminuye a $8,4 \%$ en áreas rurales (DANE, 2013).

Estas asimetrías regionales, así como la urgencia de contener la ampliación de la brecha digital, reclaman una intervención decidida por parte del Estado. Sin embargo, el marco que delimita la política de telecomunicaciones, establecido por la Ley 1341 de 
2009 "Por la cual se definen principios y conceptos sobre la sociedad de la información y la organización de las Tecnologías de la Información y las Comunicaciones (TIC), se crea la Agencia Nacional de Espectro y se dictan otras disposiciones", ha privilegiado la promoción de políticas de acceso universal que buscan garantizar que en ausencia de oferta privada de servicios, exista al menos un punto de acceso disponible para la comunidad, en desmedro de metas más ambiciosas orientadas hacia la universalización del servicio que buscan la penetración de servicios en cada hogar. Esta inclinación representa una pérdida de eficiencia en la inversión de recursos y una dilatación del proceso de difusión tecnológica debido a que la financiación de costos operativos de la instalación y puesta en servicio de las soluciones de acceso comunitario tiene un alto costo de oportunidad si se comparan los resultados asociados al despliegue de infraestructura de redes de transporte y acceso.

Dada la estrecha relación entre las condiciones de acceso y uso de la conectividad, las intervenciones públicas en materia de difusión tecnológica requieren complementar, de manera simultánea, instrumentos del lado de la oferta (i.e. marco regulatorio para la promoción de competencia, adecuación de condiciones de acceso, entre otras) y de demanda (i.e. asequibilidad del servicio y bienes complementarios, generación de contenidos, alfabetización digital y demás estímulos a la apropiación tecnológica). Esta medida, identificada como uno de los criterios orientadores en el manual de estrategias de banda ancha del Banco Mundial (Kelly y Rossotto, 2012), torna problemática la optimización de recursos y exige soportar la toma de decisiones con la construcción de diagnósticos confiables y oportunos.

En el caso de Colombia, el Plan Vive Digital, formulado por el Ministerio TIC en respuesta a los lineamientos previstos en el Plan Nacional de Desarrollo 2010-2014, contempló el diseño de un ecosistema digital que integra instrumentos de oferta (infraestructura y servicios) y demanda (aplicaciones y usuarios). La meta general del plan consiste en multiplicar por cuatro el número de conexiones a Internet, pasando de 2.2 millones de suscriptores - registrados como línea base en 2010- a 8.8 millones en 2014. Si al primer trimestre de 2013 la penetración de Internet alcanzó los 6 millones, se espera que la meta se supere exitosamente al término de la presente administración.

No obstante, valorar el impacto efectivo de cada uno de los componentes del ecosistema digital en la difusión tecnológica no es sencillo, y la persistencia de las asimetrías regionales advierte la urgencia de aplicar criterios más equitativos de inclusión digital. En cumplimiento de las metas establecidas por el plan, en 2011 en el segmento de infraestructura se emprendió el Proyecto Nacional de Fibra Óptica mediante la ejecución de un contrato de aporte financiado con recursos del Fondo TIC y adjudicado al operador Azteca Comunicaciones. Esta iniciativa estima ampliar la red troncal de fibra óptica en 753 cabeceras municipales del país. Ahora bien, las zonas que quedaron excluidas del proyecto, pertenecientes a los departamentos del Chocó y territorios nacionales en las 
regiones de la Amazonía y Orinoquía, continúan demostrando la existencia de fallas de mercado que resulta necesario corregir.

Problemáticas similares en otros países han abierto una intensa discusión teórica acerca de la posibilidad de clasificar la conectividad como un bien público (Frischmann, 2005). Desde la economía clásica, la identificación de esta categoría de bienes permitió conciliar las tensiones entre el interés individual y el interés público, a través de la intervención correctiva del Estado. Esta tradición del pensamiento anglosajón fue objetada por la Escuela Historicista Alemana de Economía en el Siglo XIX, cuyos precursores -Roscher, Hildebrand y Knies-, inspirados en los preceptos filosóficos de List (1857), desarrollaron un marco teórico en el que el comportamiento de los individuos no sólo se orienta por el egoísmo sino también por un sentido de comunidad (Gemeinsinn). De esta forma, el Estado deja de concebirse como mecanismo de corrección para entenderse como un agente de propósitos comunes, y la disyuntiva entre el mercado y la esfera pública es remplazada por una relación de interdependencia y complementariedad (Sturn, 2010).

Este debate condujo años más tarde al surgimiento de la teoría del intercambio voluntario de las finanzas públicas, sintetizado por Musgrave (1939) como el propósito de explicar el proceso de la tributación y gasto público a partir de las mismas leyes que rigen la determinación del valor y los precios en el mercado. En este orden de ideas, los impuestos aparecen como contribuciones voluntarias a cambio de los bienes y servicios provistos por el Estado. Por su parte, las negociaciones políticas que influyen en la distribución del gasto público, asumiendo que el poder de los participantes es el mismo, conducen a un resultado óptimo en la asignación de recursos. Este enfoque tuvo importantes implicaciones en los estudios sobre la justicia y optimización del sistema tributario y antecedió la elaboración de teorías orientadas a diferenciar con mayor claridad la naturaleza de los bienes públicos y privados.

En los años cincuenta, Samuelson (1954) formuló las condiciones de ineficiencia que impiden al mercado alcanzar un óptimo de Pareto en presencia de los bienes públicos puros. La primera consiste en que el consumo individual de ese bien no ocasiona una disminución en el consumo que realicen otros individuos. En otras palabras, el consumo total del bien público es igual al consumo que realiza cada individuo; esto significa que un bien público no genera rivalidad en su consumo. Por el contrario, cuando se trata de un bien privado, el consumo de éste es igual a la suma del consumo de cada individuo, lo que implica que su consumo genera rivalidad. En un mercado de bienes privados, la cantidad óptima de producción es aquella en la que, a un precio que corresponde al costo marginal de producción, cada consumidor obtiene un beneficio marginal que excede el precio. En el caso de los bienes públicos el punto óptimo está dado por la suma de los beneficios marginales que iguala el costo marginal de producir el bien. Por tanto, los individuos pagarán un impuesto que equivalga al beneficio que les reporta 
el consumo del bien público. Esta condición desborda la capacidad de los mercados porque éstos ofertan bienes a un mismo precio sin diferenciar el beneficio marginal de cada consumidor.

La segunda condición se refiere a la imposibilidad de excluir a cualquier individuo en la oferta del bien público. Una vez provisto, cualquier individuo puede acceder al consumo del bien, aún sin contribuir a su financiación. Esto se debe a que el consumo de un individuo adicional no implica ningún incremento en el costo de producción. Esta circunstancia impide conocer las preferencias de los consumidores y asignar una tasa de contribución efectiva. Del mismo modo, la ausencia de incentivos para contribuir a la financiación del bien, según la lógica del free-rider, dificulta estimar la cantidad óptima de su respectiva producción. Esta clasificación, propuesta para diferenciar los bienes públicos y privados, ha motivado toda serie de discusiones en relación con la capacidad del mercado para proveer bienes públicos y la capacidad del Estado para proveer bienes privados, las cantidades óptimas de producción, los factores determinantes de la acción colectiva y los posibles métodos para cuantificar las tasas impositivas requeridas para la financiación de los bienes públicos.

De acuerdo con lo anterior, la literatura económica de los bienes públicos impuros ha proporcionado un complemento al estudio de las fallas de mercado. En su teoría de los bienes Club, Buchanan (1965) ilustró cómo un bien público susceptible de exclusión pero que no genera rivalidad en su consumo, manteniendo bajos niveles de congestión, puede ser provisto en condiciones de eficiencia sin requerir la intervención del Estado. Esto se explica por la posibilidad de aprovechar economías de escala en la producción del bien, de tal modo que en la medida en que aumenta el número de miembros, el costo promedio de producir el bien se reduce. La cantidad producida del bien se determina en relación con el nivel de congestión, del que depende el beneficio individual del consumidor.

Desde otro ángulo, la posibilidad del mercado de producir bienes públicos de manera eficiente ha sido reivindicada con base en los incentivos provistos por la estructura institucional. A través del recuento descriptivo sobre el sistema privado de administración de faros en Gran Bretaña, Coase (1974) refutó la idea de que este servicio, empleado en los trabajos de numerosos economistas del bienestar como ejemplo típico de un bien público, demanda la intervención directa del Estado. El éxito de dicho sistema, preservado sin necesidad de acudir al recaudo de impuestos, según el autor, ha consistido en la definición de los derechos de propiedad y los mecanismos legales establecidos para hacerlos cumplir.

En contravía con la propuesta de delimitar la frontera entre el mercado y la intervención estatal a partir de la naturaleza de los bienes públicos, Musgrave (1959) definió una categoría adicional de bienes que, sin ser públicos, demandan la intervención del Estado a saber: los bienes de mérito. Éstos se caracterizan por presentar exclusión y/o rivalidad, lo que significa que pueden ser ofrecidos por el mercado pero teniendo en 
cuenta que los consumidores no siempre toman decisiones racionales y suficientemente informadas, por razones de bienestar, deben ser provistos por el Estado. El autor señala como ejemplos la educación, los subsidios de vivienda y los sistemas de salud. Aunque esta clase de bienes ha sido acogida en teorías distributivas, como la Teoría de la Justicia de Rawls (Ege y Igersheim, 2010), ha sido objeto de fuertes cuestionamientos por parte de la corriente ortodoxa. De acuerdo con McLure (1968) esta teoría trasgrede la soberanía del consumidor, principio básico de la economía normativa. En estos términos, cualquier objetivo que deba perseguir el Estado mediante el gasto público, debe obedecer a la satisfacción de los deseos de los consumidores tan eficientemente como sea posible.

En años recientes ha surgido un enfoque teórico, liderado por Ostrom (1990), que busca superar la frontera entre el Estado y el mercado a través de un modelo de co-producción en el que los ciudadanos participan en la provisión de bienes y servicios. Esta propuesta plantea que el comportamiento observado en la administración de bienes comunes subvierte la lógica de la acción colectiva ilustrada por Hardin (1968) para señalar que los hombres en busca de su propio interés hacen un uso irracional de los bienes comunes, generando la ruina colectiva. Desde esta perspectiva, las normas sociales y el conjunto de incentivos juegan un rol determinante en la gestión autónoma de las comunidades.

A la luz de las precisiones anteriores, es posible observar que en el mercado de la conectividad están presentes las condiciones de rivalidad y exclusión en el consumo del servicio. A medida que hay mayor cantidad de usuarios en la red, y que el uso de la conectividad de cada uno de ellos demanda más capacidad de trasmisión, aumenta el nivel de congestión. De otro lado, las diversas soluciones tecnológicas permiten excluir el acceso al servicio sin incurrir en costos significativos por ese control. Lo anterior impide reconocer el servicio de conectividad como un bien público. Por consiguiente, abordar la provisión del mismo como uno de mérito es la opción llamada en su reemplazo (Locksley, 2011). En este caso, desde el punto de vista operativo surgen numerosos interrogantes sobre la viabilidad financiera y técnica de implementar subsidios cruzados, o constituir empresas públicas que compitan, en condiciones de mercado, con operadores privados para ampliar la cobertura en zonas identificadas como brechas puras. Resta acotar que la alternativa de abordar la conectividad como un bien común, provisto bajo esquemas de gestión colectiva, se aleja de la realidad que viven muchas zonas aisladas con elevados niveles de pobreza, complejidad geográfica, alta dispersión poblacional, fragilidad institucional y precario capital social, debido a la gran inversión que requiere el despliegue, operación y mantenimiento de infraestructura.

\section{Consideraciones finales}

En principio, la imperiosa necesidad de llevar a cabo una inclusión digital que permita generar igualdad de oportunidades, garantizar derechos y libertades políticas, y mejorar la 
calidad de vida de los ciudadanos, unida a las ventajas económicas que conlleva la difusión tecnológica, motiva la elección de una intervención estatal directa. Ante la imposibilidad de concederle a la conectividad el tratamiento de un bien público, y considerando los riesgos asociados con la prestación del servicio por parte de una empresa pública que deba competir en igualdad de condiciones con operadores privados, cabe contemplar como escenario alternativo un modelo de intervención pública indirecta. En este caso, acoger obligaciones de servicio universal para que todos los operadores del mercado presten el servicio a quien lo demande, sin discriminación alguna, es una salida promisoria. Esta medida exige analizar los resultados que haya arrojado su implementación en otros países que compartan características similares a las colombianas y el impacto que podría generar en el mercado de telecomunicaciones, en virtud de las dinámicas de competencia existentes.

En síntesis, la pertinencia y viabilidad de las políticas de difusión tecnológica a través de las cuales se promueva la inclusión digital, deberán someterse a un análisis exhaustivo que aborde las características más relevantes del marco institucional del sector de las telecomunicaciones. Elementos tales como la trayectoria de la regulación, la estructura de la competencia, la capacidad técnica y operativa de las entidades públicas del sector y los lineamientos estratégicos vigentes, son algunos ejemplos. Por último, la visibilidad de la brecha digital y la relación de fuerzas entre los grupos de interés son partes esenciales de la construcción del diagnóstico.

\section{Referencias Bibliográfícas}

Adachi, F. (2002). Evolution Towards Broadband Wireless Systems. IEEE, 1, 19-26.

Al-Jaghoub, S., Al-Ahliyya, A. y Westrup, C. (2009). Reassessing social inclusion and digital divides. Journal of Information, Communication \& Ethics in Society, 7 (2/3), 146-158.

Balkin, Jack. (2004). Digital Speech and Democratic Culture: A Theory of Freedom of Expression for the Information Society. New York University Law Review, 79 (1), 1-55. Baudrillard, J. (2007). Cultura y Simulacro. Barcelona: Kairos.

Bell, D. (1991). El advenimiento de la sociedad post-industrial. Madrid: Alianza.

Benkler, Y. (2000). From Consumers to Users: Shifting the Deeper Structures of Regulation Toward Sustainable Commons and User Access. Federal Communications Law Journal, 5 (3), 561-579.

Birdsall, W. (2011). Human capabilities and information and communication technology: the communicative connection. Ethics \& Information Technology, 13, 93-106.

Blackman, C. y Forge, S. (2008). The future of universal service in Europe. Info, 10 (5/6), 152-165. 
Bouwman, H. y Fijnvandraat, M. (2006). Flexibility and broadband evolution. Telecommunications Policy, 30, 424-444.

Buchanan, J. M. (1949). The pure theory of government finance: A suggested approach. Journal of Political Economy, 57, 496-506.

Buchanan, J. M. (1965). An economic theory of clubs. Economica, 32, 1-14.

Buchanan, J. (1967). Public Finance in Democratic Process. Chapel Hill: University of North Carolina Press.Caldow, J. (2004). e-Democracy: Putting Down Global Roots. Washington, DC: Institute for Electronic Government, IBM.

Calvo, A. (2012). Universal Service Policies in the Context of National Broadband Plans. OECD Digital Economy Papers, 203.

Cambini, C. \& Jiang, Y. (2009). Broadband investment and regulation: A literature review. Telecommunications Policy, 33, 559-574.

Castells, M. (2002). The Information Age: Economy. Society and Culture. I: The Rise of the Network Society. Oxford: Blackwell Publishing.Castells, M. (2009). Comunicación y poder. Madrid: Alianza Editorial.

Coase, R. (1974). The Lighthouse in Economics. Journal of Law and Economics, 17 (2), 357-376.

Cohen, R. (2011). Internet History. International Journal of Technoethics, 2 (2), 45-64.

Crandall, R. (2003). Broadband Communications. Washington: Brookings Institution.

Crawford, S. (2013). Captive audience: the telecom industry and monopoly power in the new gilded age. New Haven: Yale University Press.

Cullis, J. y Jones, P. (1987). Microeconomics and the Public Economy: A Defense of Leviathan. Oxford: Blackwell Publishers.

DANE (2013). Encuesta TIC 2012. Bogotá: DANE. Disponible en: http://www.dane. gov.co/index.php/tecnologia-e-innovacion-aliasD’Halluin, Y., Forsyth, P. y Vetzal, H. (2002). Managing Capacity for Telecommunications Networks under Uncertainty. Journal IEEE/ACM, 10 (4), 579-587.

DiMaggio, P., Harggitai, E., Neuman, R. y Robinson, J. (2001). Social implications of the Internet. Annual Review of Sociology, 27, 307-36.Ege, R. y Igersheim, H. (2010). Rawls's justice theory and its relations with the concept of "public good". The European journal of the history of economic thought, 17 (4), 1001-1030.

Faulhaber, G. (2010). Innovation in the Wireless Ecosystem: A Customer-Centric Framework. International Journal of Communication, 4, 73-112. 
Faulhaber, G. y Hogendorn, Ch. (2000). The market structure of broadband telecommunications. The Journal of Industrial Economics. 48 (3), o022-1821.

Florenzano, M. (2010). Government and the Provision of Public Goods: From Equilibrium Models to Mechanism Design. The European Journal History of Economic Thought, 17 (4), 1047-1077.

Frischmann, B. (2005). An Economic Theory of Infrastructure and Commons Management. Minnesota Law Review, 89, 917-1029.

Hardin, G. (1968). The Tragedy of the Commons. Science, 162, 1243-12567.

Hilbert, M. (2011). The end justifies the definition: The manifold outlooks on the digital divide and their practical usefulness for policy-making. Telecommunications Policy, 35 (8), 715-736.

Hofmokl, J. (2010). The Internet Commons: Towards an Eclectic Theoretical Framework. International Journal of the Commons, 4, 226-250.

Horrigan, J. (2011). What are the consequences of being disconnected in a BroadbandConnected world? American Academy of Art \& Sciences, 140 (4), 17-31.

International Telecommunication Union (ITU) (2013). ICT Statistics. Ginebra: ITU. Disponible en: http://www.itu.int/en/ITU-D/Statistics/Pages/stat/default.aspx

Jordán, V. y De león, O. (2010). "La banda ancha y la concreción de la revolución digital”. En: V. Jordan, H. Galperin, W. Peres (comps), Acelerando la revolución digital: Banda Ancha para América Latina y el Caribe (pp. 13-49). Santiago de Chile: CEPAL.

Kelly, T. y Rossotto, C. (2012). Broadband Strategies Handbook. Washington: World Bank Group.

Keniston, K., y Kumar, D. (2003). The Four Digital Divides. Delhi: Sage.

Kumar, A. (2010). Evolution of Mobil Wirless Communication Networks: $1 \mathrm{G}$ to $4 \mathrm{G}$. International Journal of Electronics \& Communication Technology, 1 (1), 68-72.

Kurz, A. y Neyman, A. (1983). Voting for Public Goods. The Review of Economic Studies, $50(4), 677-693$.

Laffont, J., Marcus, S., Rey, P. y Tirole, J. (2003). Internet interconnection and the offnet-cost pricing principle. The RAND Journal of Economics, 34 (2), 370-390.

Lessig, L. (2001). The future of ideas. The fate of the commons in a connected world. Nueva York: Random House.

Ley 1341 de 2009, "Por la cual se definen principios y conceptos sobre la sociedad de la información y la organización de las Tecnologías de la Información y las 
Comunicaciones -TIC-, se crea la Agencia Nacional de Espectro y se dictan otras disposiciones". Bogotá, Diario Oficial 47426 de julio 30 de 2009.

Locksley, G. (2011). State Aid and Broadband, a response to DG Competition. European Commission Report. Disponible en: http://ec.europa.eu/competition/consultations/2011_broadband_guidelines/locksley_gareth_en.pdf.Mansell, R. (2009). The information society. Critical concepts in sociology. Londres: Routledge.

Mason, R. (2000). Network externalities and the Coase conjecture. European Economic Review, 44 (10), 1981-1992.

Masuda, Y. (1984). La sociedad informatizada como sociedad postindustrial. Madrid: Tecnos.

McLure, C.E. (1968). Merit Wants: A Normative Empty Box. Finanzarchiv, 27 (2), 474-483.

Medema, S. (2009). The Hesitant Hand: Taming Self-Interest in the History of Economic Ideas. Nueva York: Princeton University Press.

Melody, W. H. (1990). The Information in Information Technology. Where Lies the Public Interest. Intermedia, 18 (3), 10-17.

Ministerio de Tecnologías de la Información y las Comunicaciones (Min TIC).(2013).Boletín SectorialdelasTIC.Bogotá:MinTIC.Disponibleen:http://colombiatic.mintic.gov.co/ index.php/novedades/124-boletin-trimestral-de-las-tic-primer-trimestre-de-2014.

Musgrave, R. A. (1939). The Voluntary Exchange Theory of Public Economy. The Quarterly Journal of Economics, 53 (2), 213-237.

Musgrave, R. A. (1959). The Theory of Public Finance. Nueva York: McGraw-Hill Book Company.

Norris, P. (2001). Digital Divide. Civic Engagement, Information Poverty, and the Internet Worldwide. Cambridge: Cambridge University Press.

Organisation for Economic Co-operation and Development. (2001). Understanding the Digital Divide. París: OECD.

Oosterlaken, I., Van den Hoven, J., Kandachar, P., \& Mani, M. (2009). Technology and human development: A capability approach. Delft: University of TechnologyIndian Institute of Science.

Organización de Naciones Unidas - ONU (2012). Promoción, protección y disfrute de los derechos humanos en Internet, A/HRC/20/L.13. Nueva York: ONU-Consejo de Derechos Humanos.

Ostrom, E. (1990). Governing the Commons: The Evolution of Institutions for Collective Action. Cambridge: Cambridge University Press. 
Ostrom, E. y Walker, J. (1997). Neither markets nor states: Linking transformation processes in collective action areas. En: D. C. Mueller (Ed.), Perspectives on Public Choice (pp. 35-72). Cambridge: Cambridge University Press.

Preston, P. (2001). Reshaping communications: technology, information and social change. Londres: Sage.

Samuelson, P. (1954). The Pure Theory of Public Expenditure. The Review of Economics and Statistics, 36 (4), 387-389.

Sarkar, M., Cavusgil, T. \& Aulakh, P. (1999). International Expansion of Telecommunication Carriers: The Influence of Market Structure, Network Characteristics, and Entry Imperfections. Journal of International Business Studies, 30 (2), 361-381.

Solum, L. y Chung, M. (2003). The Layers Principle: Internet Architecture and the Law. Public Law and Legal Theory, Research Paper 55. University of San Diego. Disponible en: http://ssrn.com/abstract $=416263$.

Stehr, N. (1994). Knowledge Societies. Londres: Sage.

Steinmueller, W. (2000). Will new information and communication technologies improve the 'codification' of knowledge? Industrial and Corporate Change, 9 (2), 361-376.

Sturn, R. (2010). Public Goods' before Samuelson: Interwar Finanzwissenschaft and Musgrave's Synthesis. The European Journal History of Economic Thought, 17 (2), 279-312.

Teppayayon, O. y Bohlin, E. (2010). Broadband Universal Service in Europe: A Review of Policy Consultations 2005-2010. Communications \& Strategies, 80, 21-37.

Toffler, A. (1981). La Tercera Ola. Bogotá : Plaza \& Janés.

Van den Hoven, J. y Rooksby, E. (2008). Distributive Justice and the Value of Information. A (Broadly) Rawlsian Approach. En: Van den Hoven, J. y Weckert, J. (Eds.). Information Technology and Moral Philosophy, Cambridge: Cambridge University Press, pp. 376-395.

Warschauer, M. (2003). Technology and social inclusion: Rethinking the digital divide. Cambridge: MIT Press.

Weber, S. (2004). The Success of Open Source. Cambridge: Harvard University Press.

Webster, F. (2006). Theories of the information society. Nueva York: Routledge. 